\title{
Challenges of Total Endovascular Repair of Chronic Type B Aortic Dissection
}

\author{
Mohamad Hamady ${ }^{1,2}$ (1) C Colin Bicknell ${ }^{2,3}$
}

Received: 9 July 2020/ Accepted: 8 August 2020/Published online: 7 September 2020

(C) Springer Science+Business Media, LLC, part of Springer Nature and the Cardiovascular and Interventional Radiological Society of Europe (CIRSE) 2020

Chronic post-dissection thoracoabdominal aortic aneurysm (pD-TAAA) is a significant challenge to manage for open, hybrid or endovascular approaches. The main potential anatomical challenges confronting total endovascular approach include: short proximal landing zone, severe aortic angulation, tight compression of true lumen with or without thrombosed false lumen, target vessel take-off from false lumen, hostile take-off angle of target vessel and extension of dissection flap deeply into target vessel or iliac arteries.

Early and contemporary experience including ours at Imperial College reports technical and clinical outcome success for total endovascular intervention [1]. Verzini et al. in the CVIR issue published online in June 2020 [2] have demonstrated their multicentre experience with custom-made f/brEVAR stent grafts using one manufacturer (Cook Medical, Brisbane, Australia) in 21 patients with pdTAAA. We congratulate Verzini's team for this excellent work, with a well-described technical description. Especially important is the authors' sage advice that careful and comprehensive planning together with cohesive teamwork is mandatory.

Anatomical challenges are of paramount importance. The compression of true lumen remains one of the biggest

\section{Mohamad Hamady}

m.hamady@imperial.ac.uk

1 Department of Interventional Radiology, St Mary's Hospital, Praed Street, Paddington, London W2 1NY, UK

2 Department of Surgery and Cancer, Imperial CollegeLondon, London W2 1NY, UK

3 Regional Vascular Unit, St Mary's Hospital, Praed Street, Paddington, London W2 1NY, UK reasons for concern during planning stage. Three options are available including pre-stenting of the thoracic aorta, penetration of the flap using the sharp end of the wire, reentry device or TIPPS needle, or visceral hybrid revascularisation. We have experience with all these options. The issue with TEVAR pre-stenting is no accurate prediction of diameter expansion of the compressed true lumen. It is conceivable that the intimo-medial flap becomes thicker and more rigid in chronic phase of dissection [3]. Puncturing intimo-medial flap with the sharp end of wire is not really a controlled manoeuvre. We find the use of a re-entry device is a safe, successful and better controlled approach. In young patients and/or those with connective tissue disease, in particular with awkward anatomy, visceral hybrid revascularisation appears the most technically successful and durable strategy [4]. There are, however, significant morbidity and mortality and needs excellent open surgical skills.

The development of inner branch stent graft (TAMBE, Gore \& Associates, Flagstaff, Arizona) (E-nside, JOTEC, Cryolife, Germany) is a promising technology. Our initial experience with JOTEC E-nside, inner branch device is encouraging.

For creating adequate proximal landing zone, we are liberal in using custom-made scallop and fenestration thoracic stent graft to accommodate the left subclavian artery or the innominate artery (Fig. 1). We believe that this approach further minimises the physiological insult to the patients [5].

The main criticism of endovascular techniques is durability, and of course, endoleak is the most important factor affecting durability of the total endovascular approach in patients with chronic dissection. The incidence of significant endoleak ranges from 14 to $66 \%[1,6]$. Besides issues 
Fig. 1 Patient with aneurysmal aorta post-complex chronic aortic dissection treated with scallop to innominate artery, carotid-carotid and carotidsubclavian bypass and visceral hybrid repair. A and B Volume rendering (VR) and maximum intensity projection $\mathrm{CT}$ scan shows compressed true lumen (arrow), left renal artery supplied by false lumen (thick arrow) and metallic aortic valve (star). C VR CT scan shows scallop thoracic stent graft (thin arrow) and visceral surgical bypasses (thin arrows)
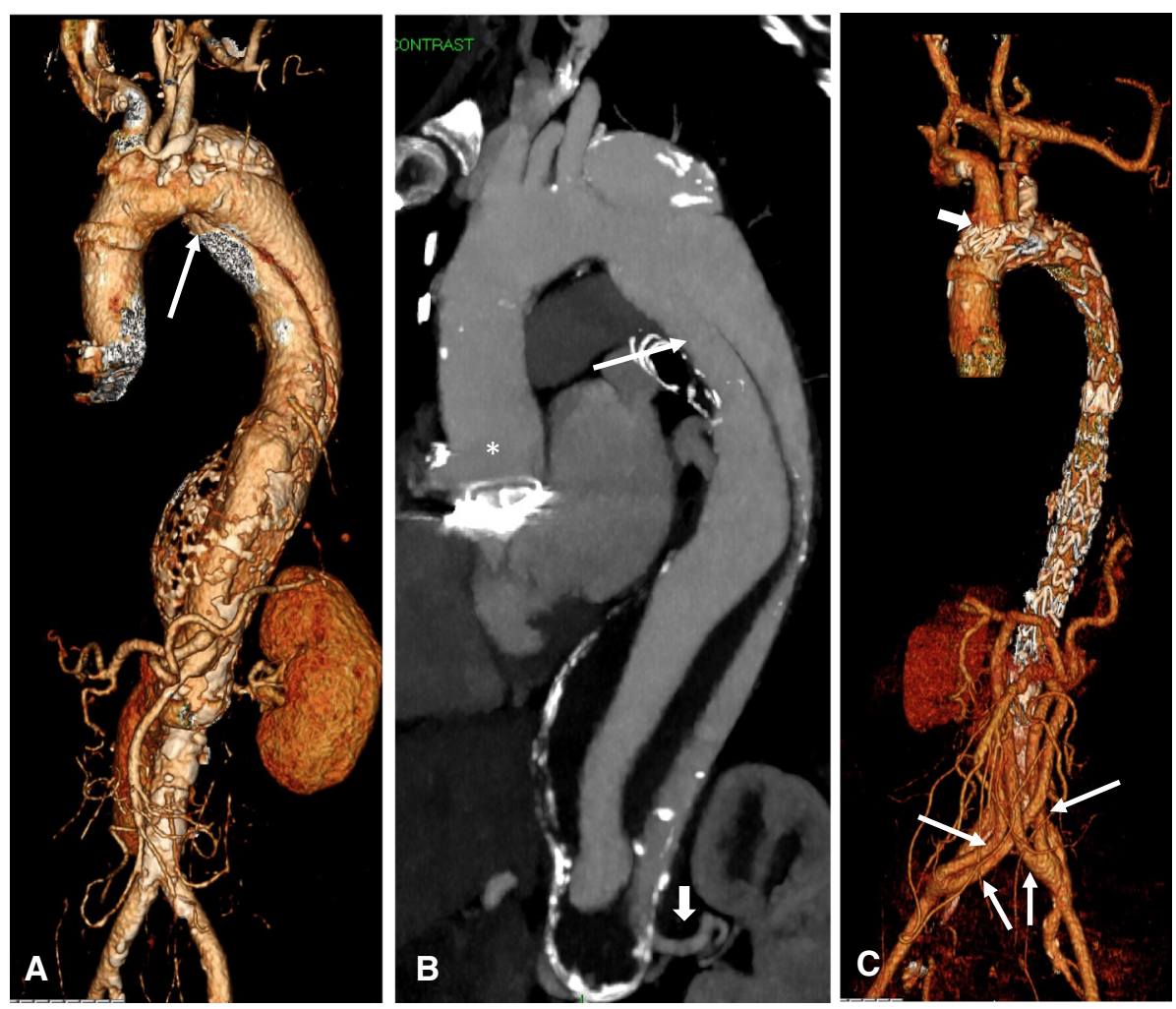

affecting immediate technical issues, two main factors can play a significant role. The first is the compliance of the bridging stents and the second is the hemodynamic and morphological changes in dissected aorta following successful stenting, thrombosis of false lumen and subsequent shrinkage of aneurysm sac. Rigidity, conformability, stability and kink resistance are critical features. It is yet to be seen if the new technology, such as Viabahn VBX (Gore \& Associates, Flagstaff, Arizona) will fill the gap.

There are equally many unanswered clinical management questions. For example, paraplegia is a devastating complication post-aortic interventions. The extensive coverage of the aorta is a leading cause of spinal cord ischaemia [7]. The authors have confirmed our experience with staging approach as a valid option to reduce the risk of ischaemia. However, further research is needed to establish the exact staging time, sequence of component assembly as well as a validated and practical neurological monitoring.

Verzinis et al. describe excellent short- and midterm results. Optimal endovascular and clinical strategies are yet to be determined to secure long-term success.

Funding No funding is attached to this paper.

\section{Compliance with Ethical Standards}

Conflict of interest $\mathrm{MH}$ has received research grants from Cryolife and Gore \& Associates and a speaker honorarium from Terumo Aorta and Gore \& Associates. CB has received a speaker honorarium from Medtronic, Gore \& Associates and Terumo Aorta and Education grants from Gore and Terumo Aorta.

Ethical Approval All procedures performed in studies involving human participants were in accordance with the ethical standards of the institutional and/or national research committee and with the 1964 Helsinki Declaration and its later amendments or comparable ethical standards.

Informed Consent For this type of study, informed consent for publication is not required.

Consent for Publication Consent for publication was obtained for every individual person's data included in the study.

\section{References}

1. Oikonomou K, Kasprzak P, Katsargyris A, Marques De Marino P, Pfister K, Verhoeven E. Mid-term results of fenestrated/branched stent grafting to treat post-dissection thoraco-abdominal aneurysms. Eur J Vasc Endovasc Surg. 2019;57(1):102-9.

2. Verzini F, Ferrer C, Parlani G, Coscarella C, Giudice R, Frola E, Ruffino MA, Varetto G, Gibello L. Mid-term outcomes of complex endografting for chronic post-dissection thoracoabdominal aortic aneurysms. Cardiovasc Intervent Radiol. 2020. https://doi.org/10. 1007/s00270-020-02555.

3. Peterss S, Mansour AM, Ross JA, et al. Changing pathology of the thoracic aorta from acute to chronic dissection: literature review and insights. J Am Coll Cardiol. 2016;68(10):1054-65.

4. Drinkwater SL, Böckler D, Eckstein H, et al. The visceral hybrid repair of thoraco-abdominal aortic aneurysms-a collaborative approach. Eur J Vasc Endovasc Surg. 2009;38(5):578-85. 
5. Alsafi A, Bicknell CD, Rudarakanchana N, et al. Endovascular treatment of thoracic aortic aneurysms with a short proximal landing zone using scalloped endografts. J Vasc Surg. 2014;60(6):1499-506.

6. Kitagawa A, Greenberg RK, Eagleton MJ, Mastracci TM, Roselli EE. Fenestrated and branched endovascular aortic repair for chronic type B aortic dissection with thoracoabdominal aneurysms. J Vasc Surg. 2013;58(3):625-34.
7. Drinkwater SL, Goebells A, Haydar A, et al. The incidence of spinal cord ischaemia following thoracic and thoracoabdominal aortic endovascular intervention. Eur J Vasc Endovasc Surg. 2010;40(6):729-35.

Publisher's Note Springer Nature remains neutral with regard to jurisdictional claims in published maps and institutional affiliations. 